Tropical Journal of Pharmaceutical Research September 2018; 17 (9): 1733-1738

ISSN: $1596-5996$ (print); 1596-9827 (electronic)

(c) Pharmacotherapy Group, Faculty of Pharmacy, University of Benin, Benin City, 300001 Nigeria.

\title{
1, 25-Dihydroxyvitamin D3 suppresses cell cycle progression and thus growth of prostate cancer cells by inducing expression of limb bud and heart development (LBH)
}

\author{
Heng Du ${ }^{1}$, Jing Zhuang ${ }^{2}$, Yaping Gui ${ }^{3}$, Qiuyue $\mathrm{Xu}^{4}$, Li Zhu ${ }^{5}$, Le Gao ${ }^{1}$, Jie Jiang ${ }^{5 *}$ \\ ${ }^{1}$ Department of Urology, Second Affiliated Hospital of Xinjiang Medical University, Xinjiang 860063, ${ }^{2}$ Department of Nephrology, \\ People's Hospital of Xinjiang Uygur Autonomous Region,Urumqi, 830000, ${ }^{3}$ Tongji Hospital Affiliated Tongji University in \\ Shanghai, Shanghai, 200000, ${ }^{4}$ Nanjing University of Chinese Medicine, Nanjing 210023, ${ }^{5}$ Department of Urology, Affiliated \\ Hospital of Changchun University of Traditional Chinese Medicine, Changchun, 130021, China
}

*For correspondence: Email: jiangjiemedcch@163.com

Sent for review: 17 March 2018

Revised accepted: 26 August 2018

\begin{abstract}
Purpose: To investigate the function of limb bud and heart development (LBH) in 1a, 25dihydroxyvitamin D3 (1,25D)-mediated inhibitory effect on proliferation of prostate cancer cells.

Methods: The inhibitory effect of 1,25D on growth and cell cycle progression of lymph node carcinoma of the prostate (LNCaP) cells was determined using cell counting kit-8 (CCK-8) assay, 5-ethynyl-2'deoxyuridine (EdU) assay and flow cytometry, while the expression levels of $L B H$ in response to treatment with $1,25 D$ were determined by quantitative reverse-transcription PCR (qRT-PCR) and western blottingting. The expression levels of $\mathrm{LBH}$ in cells were down/up regulated by transfection with siRNA or overexpression plasmids, and then cell growth and cell cycles were measured using the CCK8 assay, EdU assay, and flow cytometry. Finally, the growth inhibitory effect of 1,25D on LBH knockdown cells were determined using CCK-8 and EdU assays.

Results: Treatment with 1,25D arrested $L N C a P$ cells in G0/G1 phase of cell cycle, suppressed the growth of the cells and induced the expression of $L B H$. Overexpression/knockdown of $L B H$ in $L N C a P$ cells suppressed/promoted cell growth and accumulated/decreased cells in the G0/G1 phase. Moreover, knockdown of $L B H$ reversed the inhibitory effect of 1,25D on cell proliferation of $L N C a P$ cells. Conclusion: Inhibitory effect of $1,25 D$ on cell cycle progression and cell proliferation might be via $L B H$.
\end{abstract}

Keywords: Cell proliferation, Limb bud and heart development, Prostate cancer, 1a, 25Dihydroxyvitamin D3

\begin{abstract}
This is an Open Access article that uses a funding model which does not charge readers or their institutions for access and distributed under the terms of the Creative Commons Attribution License (http://creativecommons.org/licenses/by/4.0) and the Budapest Open Access Initiative (http://www.budapestopenaccessinitiative.org/read), which permit unrestricted use, distribution, and reproduction in any medium, provided the original work is properly credited.

Tropical Journal of Pharmaceutical Research is indexed by Science Citation Index (SciSearch), Scopus, International Pharmaceutical Abstract, Chemical Abstracts, Embase, Index Copernicus, EBSCO, African Index Medicus, JournalSeek, Journal Citation Reports/Science Edition, Directory of Open Access Journals (DOAJ), African Journal Online, Bioline International, Open-J-Gate and Pharmacy Abstracts
\end{abstract}

\section{INTRODUCTION}

1,25-Dihydroxyvitamin D3, the biologically active form of vitamin $\mathrm{D} 3$, is the ligand for the vitamin $D$ receptor (VDR) that is widely expressed in epithelial cells, including in prostate tissues and in prostate cancer [1]. Although whether 1,25D could reduce the incidence and mortality of prostate cancer is still controversial according to the results of epidemiological studies, it is well- 
known that treatment with 1,25D could alter growth and cell cycle of prostate cancer cells $[1,2]$. However, the mechanism of how $1,25 \mathrm{D}$ affects growth and cell cycle of prostate cancer cells is still not fully understood. Treatment with $1,25 \mathrm{D}$ on $\mathrm{LNCaP}$ and PC-3 cells showed that only LNCaP cells, but not PC-3 cells, were arrested in G0/G1 phase. The results suggested that 1,25D might function via p53, because LNCaP cells had wild-type of p53, whereas PC-3 cells had an inactive p53 [21,22]. However, further study using LNCaP cells with an inactivate p53 due to over-expression of a dominant-negative element of p53 showed that applying 1,25D still arrested these cells in G1 phase[3]. These results suggested that besides p53, other signaling pathways might be involved in the development of prostate cancer.

LBH encodes a highly conserved protein which functions as a tissue-specific transcription cofactor [5]. It was first identified in the embryonic limb bud and heart in mice, and further found to be expressed in various adult tissues, including the gut, peripheral nervous system, spleen, lung, kidney, bones as well as prostate [4,5]. Dysregulation of $\mathrm{LBH}$ is associated with various diseases, such as congenital heart disease, systemic lupus erythematosus, rheumatoid arthritis, as well as tumorigenesis, invasion, and metastasis [6-8]. Molecular and cellular functional studies showed that LBH was the direct target of the Wnt signaling pathway, and it could also directly function as a tumor suppresser by inducing cell cycle arrest $[9,10]$. Notably, analysis of transcription profile of proliferating RWPE1 cells under treatment with 1,25D showed that expression of LBH was upregulated in these cells [11].

In this study, the expression levels and cellular function of LBH in LNCaP cells under treatment with 1,25D were determined to find out whether LBH was involved in 1,25D-mediated regulation of cell proliferation and cell cycle of prostate cancers.

\section{EXPERIMENTAL}

\section{Cell culture and transfection}

LNCaP cells were obtained from the American Type Culture Collection (CRL-1740 TM, USA) and maintained in Dulbecco's Modified Eagle Medium (10566024, Gibco, ThermoFisher scientific, USA) with $10 \%$ fetal bovine serum. Plasmids and shRNA were transfected into cells by Lipofectamine 2000 (Invitrogen, Waltham. MA, USA).

\section{CCK-8 assay}

LNCaP cells were cultured in complete medium with different concentrations of 1,25D which was dissolved in DMSO for indicated hours in sextuplicate in 96-well plates. Then, CCK-8 reagent was added into each well. After incubation with the CCK-8 reagent for 1 hour, the absorbance at $450 \mathrm{~nm}$ was read a microplate reader (Infinite M200, Tecan Systems, San Jose, CA, USA).

\section{EdU cell proliferation assay}

Cell proliferation was measured using an EdU assay kit (C10637, Invitrogen, ThermoFisher scientific, USA). Briefly, $5 \times 10^{3}$ /well LNCaP cells were cultured in sextuplicate in 96-well plates and stimulated with different concentrations of 1,25D for $48 \mathrm{~h}$. After EdU labeling, the cells were fixed and permeabilized, and then treated with Click-iT® reaction cocktail. After a $30 \mathrm{~min}$ incubation, the nuclei were stained with DAPI and visualized using a fluorescence microscope. The EdU positive cells with fluorescence were counted.

\section{Cell cycle analysis}

Cells of each group were collected and fixed with $75 \%$ ethanol. The fixed cells were further incubated in phosphate-buffered saline (PBS) containing $0.5 \%(\mathrm{v} / \mathrm{v})$ Triton X100, $250 \mu \mathrm{g} / \mathrm{mL}$ RNase $A$ and $50 \mu \mathrm{g} / \mathrm{mL}$ propidium iodide (PI, P4170, Sigma, USA) for $30 \mathrm{~min}$ in the dark, and then the stained cells were subjected to an Accuri ${ }^{\mathrm{TM}} \mathrm{C} 6$ flow cytometer (BD Biosciences, USA) for DNA content analysis. Cell cycle distribution was calculated according to the DNA content.

\section{$q R T-P C R$}

Total RNA was extracted from the cells using TRIzol RNA isolation reagents (15596026, Invitrogen, ThermoFisher scientific, USA) following the manufacturer's protocol. The quality and quantity of the RNA samples were assessed using Nanodrop 2000 (ThermoFisher scientific, USA). The RNA $(1 \mu \mathrm{g})$ was used as template for reverse transcription through the PrimeScript ${ }^{\mathrm{TM}}$ RT reagent Kit with the gDNA Eraser (RR047Q, Takara Biomedical Technology (Beijing) Co., Ltd, China). Real-time PCR was performed on an Applied Biosystems ${ }^{\mathrm{TM}} 7500$ Fast Real-Time PCR Instrument (Applied Biosystems ${ }^{\mathrm{TM}}$, ThermoFisher scientific, USA) using SYBR® Premix Ex Taq ${ }^{\mathrm{TM}}$ II (RR820L, Takara Biomedical Technology (Beijing) Co., Ltd, China). The nucleotide 
sequences of primers used for real-time PCR were listed in Table 1:

Table 1: Primers used in real-time PCR

\begin{tabular}{ll}
\hline Primer & Sequences (5' to 3') \\
\hline LBH-sense & GCCCCGACTATCTGAGATCG \\
LBH-antisense & GCGGTCAAAATCTGACGGGT \\
$\begin{array}{l}\beta \text {-actin-sense } \\
\beta \text {-actin- }\end{array}$ & CCCTAAGGCCAACCGRGAAA \\
antisense & ACGACCAGAGGCATACAGGGA \\
&
\end{tabular}

\section{Western blotting}

Proteins were extracted in RIPA buffer and the protein concentrations of extracted proteins were determined using a Pierce ${ }^{\circledR}$ BCA Protein Assay Kit (23227, ThermoFisher Scientific, USA). Then, $10 \mu \mathrm{gg}$ extracted proteins were loaded and separated by SDSPAGE and then transferred onto PVDF membranes [20]. The membranes were then blocked in $25 \mathrm{~mL} 5 \%(\mathrm{w} / \mathrm{v})$ non-fat milk in PBS for 1 hour. Subsequently, the membrane was washed with TBST and incubated with a LBH primary antibody (ab173737; Abcam, Cambridge, UK) at $4^{\circ} \mathrm{C}$ for overnight. After washing with TBST for three times, the membranes were incubated with a secondary antibody for $45 \mathrm{~min}$ at room temperature. Finally, blots were visualized with Pierce ${ }^{\mathrm{TM}}$ ECL Western Blotting Substrate (32109, ThermoFisher Scientific, USA). GAPDH was used as an internal reference.

\section{Construction of shRNA and LBH overexpression plasmids}

Full length coding sequences of $\mathrm{LBH}$ was amplified using KOD-Plus-Neo DNA polymerase (Toyobo, Japan) with the following primers (from $5^{\prime}$ to $3^{\prime}$ ): forward: CCCGTGTCATCCTCACTCG and reverse: CAGATGCTGGCTGGTATGACC. The PCR products were ligated into a pcDNA3.1 vector (Invitrogen) and sequenced for validation. The target sequences of shRNA were designed using the BLOCK-iT ${ }^{\mathrm{TM}}$ RNAi Designer and cloned into the pLKO.1 vector. The target sequences were (from $5^{\prime}$ to $3^{\prime}$ ): shRNA1: GGATGGCCTTTCCTACCAGAT; $\quad$ ShRNA2: GGCCTTTCCTACCAGATCTTC, and shRNA3: GCCTTTCCTACCAGATCTTCC.

\section{Statistical analysis}

All data are presented as mean \pm SEM and were analyzed by Student's t test or one-way ANOVA (ANOVA), using IBM SPSS software (version 19.0, Statistical Product and Service Solutions, USA). $P<0.05$ was considered as significant difference.

\section{RESULTS}

Treatment with 1,25D upregulated the expression of LBH in LNCaP cells

Upregulation of $\mathrm{LBH}$ in the transcription profile of normal prostate RWPE1 cells under treatment with 1,25D suggested that $\mathrm{LBH}$ might be involved in 1,25D-mediated regulation of cell proliferation and cell cycle in prostate cancer cells. Thus, the inhibitory effect of $1,25 \mathrm{D}$ on LNCaP cells was confirmed data from CCK-8 assay, EdU cell proliferation assay and cell cycle analysis after treatment with $1 \mathrm{nM}, 10 \mathrm{nM}$ and $100 \mathrm{nM}$ 1,25D. As expected, 1,25D suppressed cell growth in a dose-dependent manner, as the inhibition effect increased with increasing concentration of 1,25D (Figure $1 \mathrm{~A}-\mathrm{C}$ ). And cell cycles of $\mathrm{LNCaP}$ cells were also altered by treatment with $1,25 \mathrm{D}$. Cells in G0/G1 phase increased with increasing concentration of $1,25 \mathrm{D}$, whereas the percentages of cells in G2/M plus $S$ phases were decreased (Figure 1D). The mRNA level of LBH was significantly upregulated 1 $\mathrm{h}$ after treatment with $1,25 \mathrm{D}$ and was continually induced for $48 \mathrm{~h}$ in LNCaP cells. The protein levels of LBH were also upregulated following treatment with 1,25D (Figure $1 \mathrm{E}$ and $\mathrm{F}$ ). These results indicate that treatment with $1,25 \mathrm{D}$ suppresses cell cycle progression and cell proliferation, but induced expression of LBH in LNCaP cells.

A

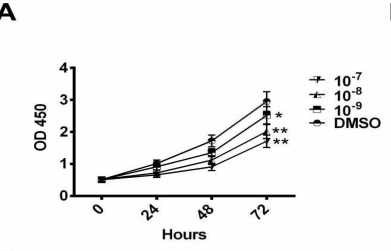

C
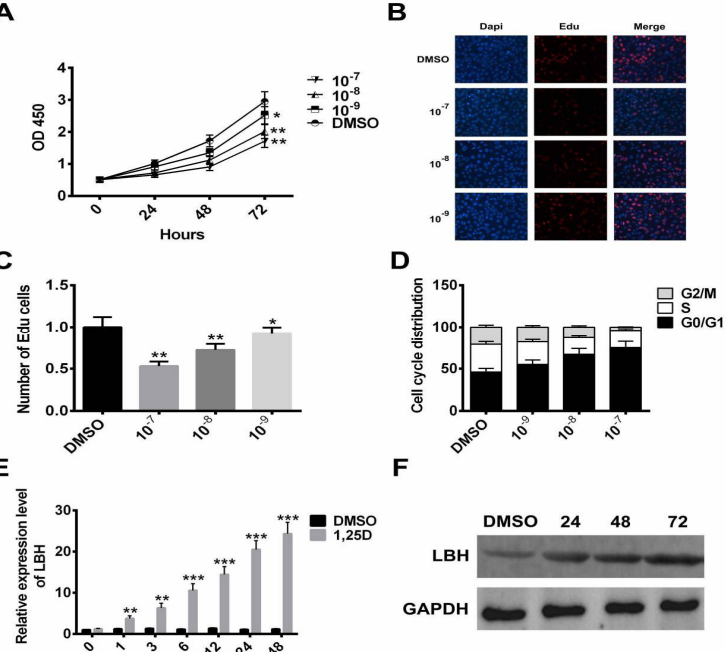

Figure 1: Treatment with 1,25D up-regulated the mRNA and protein levels of LBH. (A) Growth curves of cells treated with $1,25 \mathrm{D}$ were obtained by CCK-8 assay. (B,C) Representative fluorescent images (magnification, $\times 200$ ) of EdU assay were shown and the average numbers of EdU-labeled cells were counted. (D). Cell cycle distribution was analyzed by 
flow cytometry after PI staining. (E) Relative mRNA expression levels of LBH under treatment with $100 \mathrm{nM}$ 1, 25D were determined by qRT-PCR. (F) Relative protein expression levels of LBH under treatment with $100 \mathrm{nM} \mathrm{1,25D}$ were determined by western blotting; ${ }^{*} p<0.05,{ }^{* *} p<0.0,{ }^{* *} p<0.001$ compared with NC

\section{LBH suppressed cell proliferation and the cell cycle}

To further analyze the cellular function of LBH, shRNA-mediated knockdown plasmids and overexpression plasmids for $\mathrm{LBH}$ were constructed. Cells transfected with LBH overexpression or shRNA-mediated knockdown plasmids showed significantly higher or lower expressions of $\mathrm{LBH}$, respectively, which indicated the high efficiency of the plasmids (Figure 2).

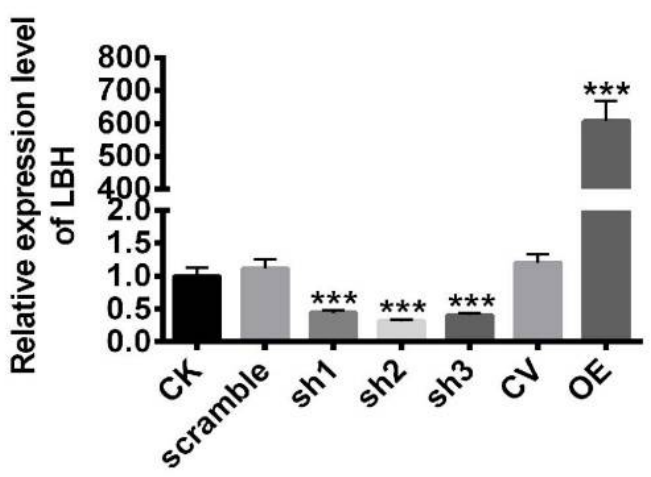

Figure 2: LBH was knocked down or overexpressed in LNCaP cells. The efficiency of shRNA and overexpression of LBH plasmids were determined by $q R T-P C R ;{ }^{* * *} p<0.001$ compared with the scramble or control vector $(\mathrm{CV})$

Then whether LBH affected cell growth was determined. Growth curves obtained using the CCK-8 assay showed that knockdown of LBH by transfection with shRNA significantly increased cell growth at $72 \mathrm{~h}$, whereas overexpression of LBH decreased the cell growth compared with both controls (scramble or CV). (Figure $3 \mathrm{~A})$. The Click-iT EdU cell proliferation assay further showed that there were more/less dividing cells in the LBH knockdown/overexpression groups when compared with control groups (scrambled/CV) (Figure 3B and C).

These results showed that cell growth was regulated by $\mathrm{LBH}$. Considering that cell growth is usually affected in the G0/G1 phase during the cell cycle, effect of LBH on the cell cycle was assessed using flow cytometry. The results showed that overexpression of LBH increased the percentage of cells accumulating in the
G0/G1 phase and decreased the percentage of cells in the $S$ phase; whereas knockdown of LBH had the opposite effect when compared with the control groups (Figure 3D). Taken together, these results showed that expression of LBH suppressed cell proliferation and delayed cell cycle at the G0/G1 phase.

A
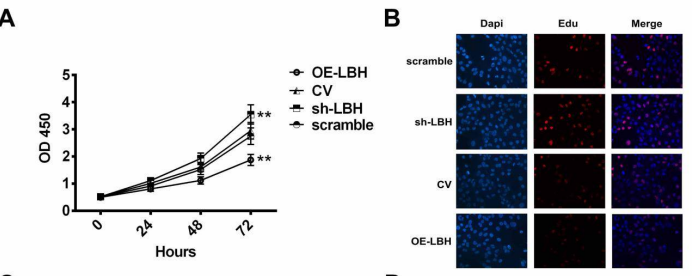

C
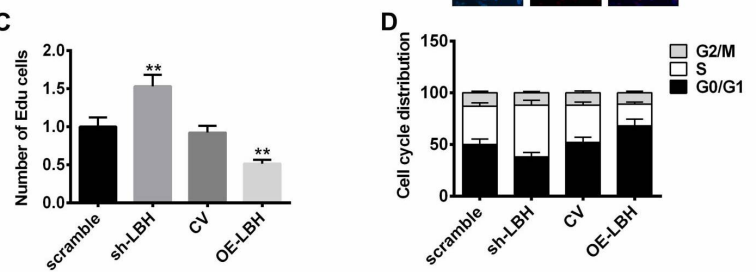

Figure 3: Expression of $\mathrm{LBH}$ regulated cell proliferation and cell cycle. (A) Growth curves of cells with knockdown (sh-LBH) or overexpression (OE-LBH) of $\mathrm{LBH}$ were analyzed by CCK-8 assay. (B,C). Representative fluorescent images (magnification, $x$ 200) of EdU assay were shown, and the average numbers of EdU-labeled cells were counted. (D) Cell cycle distributions were analyzed by flow cytometry after PI staining; ${ }^{* *} p<0.01$ compared with $\mathrm{NC}$

\section{LBH was involved in 1,25D-mediated inhibition of cell proliferation}

Since over-expression of LBH suppressed cell cycle progression and cell growth, whether inhibitory effect of 1,25D on cell cycle progression and cell growth was via inducing $\mathrm{LBH}$ was determined. If so, knockdown of LBH should eliminate or attenuate the inhibitory effect of 1,25D. Thus, cell growth of LBH knock-down cells under treatment with $1,25 \mathrm{D}$ was measured. The growth curves obtained using the CCK-8 assay showed that knockdown of LBH attenuated the inhibitory effect of 1,25D on cell growth. The growth rate of LBH knockdown cells combined with treatment with $1,25 \mathrm{D}$ was between the value for LBH knockdown cells and 1,25D treated cells (Figure 4A). EdU assay also showed similar results. The number of dividing cells of LBH knockdown cells combined with treatment with $1,25 \mathrm{D}$ was between the value for LBH knockdown cells and 1,25D treated cells (Figure $4 \mathrm{~B}$ and $\mathrm{C}$ ). These results indicate that 1,25D suppressed cell proliferation, partially via upregulating the expression of LBH. 
Knockdown of LBH hindered the inhibitory effect of $1,25 \mathrm{D}$ on cell growth.

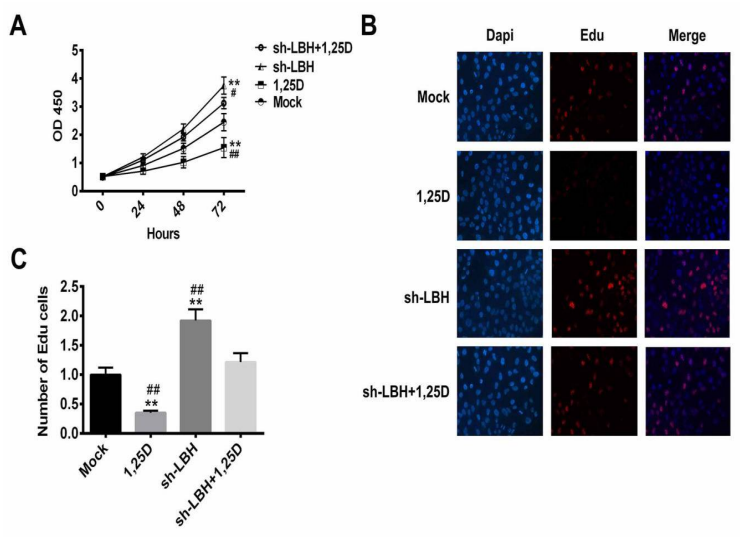

Figure 4: Knockdown of $\mathrm{LBH}$ reduced the inhibitory effect of 1,25D on cell growth. (A) Growth curves of cells were analyzed by CCK-8 assay. (B,C). Representative fluorescent images (200x) from EdU assay are shown, and the average numbers of EdUlabeled cells undergoing division were counted; ${ }^{* *} p<$ 0.01 compared with mock group; \#p < 0.05, \#\#p< 0.01 compared with sh-LBH $+1,25 \mathrm{D}$ group

\section{DISCUSSION}

Prostate cancer is one of the most commonly diagnosed cancer, and the incidence of prostate cancer was approximately 1.6 million in 2015 . It has been the second most common cause of cancer deaths in men, accounting for $29 \%$ $[12,13]$. Although most of prostate cancer usually will not affect the life expectancy of patients, the rapidly progressive metastasizing and therapyresistant prostate cancer in advanced stages is aggressive, with a poor prognosis. Traditional chemotherapy and radiation therapy only have palliative effects on these resistant cancers. Consequently, there is a need to determine the molecular pathways underlying the development and progression of prostate cancer, so as to develop more effective drugs and identify better therapeutic targets.

Limb bud and heart development (LBH) was first isolated as a novel tissue-specific transcription cofactor in mice, and it was implicated in embryonic heart development. In human breast cancer, LBH was overexpressed in aggressive basal subtype cells rather than low invasive or normal epithelial cells [5]. LBH suppressed the growth of NPC cells [9]. These results suggested that LBH might function as an anti-oncogene. The 1,25D was reported to inhibit proliferation, change the cell cycle, and stimulate apoptosis in VDR-positive cancer cells. In this study, the results showed that 1,25D regulated the cell function via LBH in prostate cancer cells.
The expression of the $L B H$ was upregulated in response to $1,25 \mathrm{D}$ in a continually induced manner was first established in this study. This result indicated that $\mathrm{LBH}$ were implicated in 1,25D-mediated regulation of cellular functioning. Then, the cellular function of LBH was determined and the results showed that expression of LBH could suppress cell proliferation and delay the cell cycle at the G0/G1 phase. Moreover, knockdown of LBH attenuated the inhibitory effects of $1,25 \mathrm{D}$ on the cell proliferation. Taken together, these results show that $\mathrm{LBH}$ were involved in 1,25D-mediated regulation of cell functions. One of the possible mechanisms is that upregulation of LBH delays cell cycle and inhibits cell proliferation via cyclins. It has been reported that cyclins, such as cyclin $D 1$, E1, and E2, were decreased by upregulation of LBH [9]. Cyclin D1 is necessary for transition from $\mathrm{G} 1$ into $S$ phase, and cyclin $\mathrm{E} 1$ and E2 activate CDK2 to drive cells from $\mathrm{G} 1$ to $S$ phase [14,15]. Moreover, decreased activity of CDK2 has also been found in vitamin D-treated prostate cancer cells [16]. Thus, LBH regulates cell proliferation might be via cyclins. However, how $L B H$ regulates $C D K s$ requires further study.

Notably, aberrantly activated $W n t / \beta$-catenin pathway is involved in various cancers, and expression of LBH is also induced by Wnt3a in breast cancer cells [10]. And 1,25D could inhibit $W n t / \beta$-catenin signaling by several mechanisms, including activation of VDR or induction of the expression of E-cadherin or the Wnt antagonist Dickkopf-1 [17-19]. Thus, it was possible that expression of LBH may be differentially regulated by several signaling pathways. And treatment with 1,25D not only inhibited $W n t / \beta$-catenin signaling, but also upregulated the expression of $\mathrm{LBH}$, both leading to inhibition of prostate cacner cell proliferation.

\section{CONCLUSION}

The results of the present study provide a better understanding of the cellular functions of LBH in prostate cancer cells. Expression of LBH suppressed cell cycle progression and cell proliferation and was induced by treatment with $1,25 \mathrm{D}$ in prostate cancer cells. 1,25D-mediated inhibitory effect on cell cycle progression and cell proliferation probably occurs by inducing expression of $\mathrm{LBH}$.

\section{DECLARATIONS}

\section{Conflict of Interest}

No conflict of interest associated with this work. 


\section{Contribution of Authors}

We declare that this work was done by the authors named in this article and all liabilities pertaining to claims relating to the content of this article will be borne by the authors. Jie Jiang designed all the experiments and revised the paper. Heng Du, Jing Zhuang and Quyue Xu performed the experiments, and Heng Du, Yaping Gui, Li Zhu and Le Gao wrote the paper.

\section{REFERENCES}

1. Maestro MA, Molnar F, Mourino A, Carlberg C. Vitamin D receptor 2016: novel ligands and structural insights. Expert Opin Ther Pat 2016; 26(11): 1291-1306.

2. Marcinkowska E, Wallace GR, Brown G. The Use of $1 \alpha$, 25-Dihydroxyvitamin D3 as an Anticancer Agent. Int $J$ Mol Sci 2016; 17(5): 729.

3. Polek TC, Stewart $L V$, Ryu EJ, Cohen MB, Allegretto EA, Weigel NL. p53 Is Required for 1,25-Dihydroxyvitamin D3-Induced Go Arrest But Is Not Required for G1 Accumulation or Apoptosis of $L N C a P$ Prostate Cancer Cells. Endocrinology 2003; 144(1): 50-60.

4. Briegel KJ, Joyner AL. Identification and characterization of Lbh, a novel conserved nuclear protein expressed during early limb and heart development. Dev Biol 2001; 233(2): 291-304.

5. Lindley LE, Curtis KM, Sanchezmejias A, Rieger ME, Robbins DJ, Briegel KJ. The WNT-controlled transcriptional regulator $L B H$ is required for mammary stem cell expansion and maintenance of the basal lineage. Development 2015; 142(5): 893-904.

6. Briegel KJ, Baldwin HS, Epstein JA, Joyner $A L$. Congenital heart disease reminiscent of partial trisomy $2 p$ syndrome in mice transgenic for the transcription factor Lbh. Development 2005; 132(14): 3305-3316.

7. Chang $Y$, Sheng $Y$, Cheng $Y$, Lin $Y$, Zhu Z, Wen L, Yang $C$, Yang L, Liu L, Zhou $F$, et al. Downregulated expression of LBH mRNA in peripheral blood mononuclear cells from patients with systemic lupus erythematosus. J Dermatol 2016; 43(1), 99-102.

8. Hammaker $D$, Whitaker JW, Maeshima $K$, Boyle $D L$, Ekwall AH, Wang W, Firestein GS. LBH Gene Transcription Regulation by the Interplay of an Enhancer Risk Allele and DNA Methylation in Rheumatoid Arthritis. Arthritis Rheumatol 2016; 68(11): 2637-2645.

9. Liu Q, Guan X, Lv J, Li X, Wang Y, Li L. Limb-bud and Heart (LBH) Functions as a Tumor Suppressor of Nasopharyngeal Carcinoma by Inducing G1/S Cell Cycle Arrest. Sci Rep 2015; 5(1): 7626-7626.

10. Rieger ME, Sims AH, Coats ER, Clarke R, Briegel KJ. The Embryonic Transcription Cofactor $L B H$ is a Direct Target of the Wnt Signaling Pathway in Epithelial
Development and in Aggressive Basal Subtype Breast Cancers. Mol Cell Biol 2010; 30(17): 4267-4279.

11. Kovalenko PL, Zhang Z, Cui M, Clinton SK, Fleet JC. 1,25 dihydroxyvitamin $D$-mediated orchestration of anticancer, transcript-level effects in the immortalized, non-transformed prostate epithelial cell line, RWPE1. BMC Genomics 2010; 11(1): 26-26.

12. Wibmer A, Burger IA, Sala E, Hricak $H$, Weber WA, Vargas HA. Molecular Imaging of Prostate Cancer. Radiographics 2016; 36(1): 142-159.

13. Global Burden of Disease Cancer Collaboration. Global, regional, and national cancer incidence, mortality, years of life lost, years lived with disability, and disabilityadjusted life-years for 32 cancer groups, 1990 to 2015: A systematic analysis for the global burden of disease study. JAMA Oncol 2016; 3: 524-548.

14. Baldin V, Lukas J, Marcote MJ, Pagano M, Draetta G. Cyclin D1 is a nuclear protein required for cell cycle progression in G1. Gene Dev 1993; 7(5): 812-821.

15. Matsumoto $Y$, Maller JL. A Centrosomal Localization Signal in Cyclin $E$ Required for Cdk2-Independent $S$ Phase Entry. Science 2004; 306(5697): 885-888.

16. Bao $B$, Hu $Y$, Ting $H$, Lee $Y$. Androgen signaling is required for the vitamin $D$-mediated growth inhibition in human prostate cancer cells. Oncogene 2004; 23(19): 3350-3360.

17. Aguilera O, Pena C, Garcia JM, Larriba MJ, Ordonezmoran P, Navarro D, Barbachano A, Silanes IL, Ballestar $E$, Fraga $M$, et al. The Wnt antagonist DICKKOPF-1 gene is induced by 1a, 25dihydroxyvitamin $D 3$ associated to the differentiation of human colon cancer cells. Carcinogenesis 2007; 28(9): 1877-1884.

18. Alvarezdiaz S, Larriba MJ, Lopezotin C, Munoz A. Vitamin D: Proteases, protease inhibitors and cancer. Cell Cycle 2010; 9(1): 32-37.

19. Larriba MJ, Gonzalezsancho JM, Barbachano A, Niell N, Ferrermayorga G, Munoz A. Vitamin $D$ is a Multilevel Repressor of Wnt/b-Catenin Signaling in Cancer Cells. Cancers 2013; 5(4): 1242-1260.

20. Maj E, Filippsurska B, Milczarek M, Psurski M, Kutner A, Wietrzyk J. Vitamin $D$ derivatives potentiate the anticancer and anti-angiogenic activity of tyrosine kinase inhibitors in combination with cytostatic drugs in an A549 non-small cell lung cancer model. Int $\mathrm{J}$ Oncol 2017; 52(2): 337-366.

21. Blutt SE, McDonnell TJ, Polek TC, Weigel NL. CalcitriolInduced Apoptosis in LNCaP Cells Is Blocked By Overexpression of Bcl-2. Endocrinology 2000; 141(1): 10-17.

22. Campbell MJ, Elstner E, Holden S, Uskokovic M, Koeffler HP. Inhibition of proliferation of prostate cancer cells by a 1 9-nor-hexafluoride vitamin D3 analogue involves the induction of p21WAF1, p27Kip1 and E-cadherin. J Mol Endocrin 1997; 19: 15-27. 